\title{
Thermal stability of gamma-irradiated polyurethane/POSS hybrid materials
}

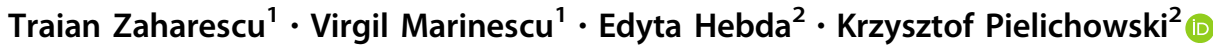

Received: 14 July 2017 / Accepted: 3 December 2017 / Published online: 11 December 2017

(C) The Author(s) 2017. This article is an open access publication

\begin{abstract}
In this work, new version: we report essential data on the stability of gamma-irradiated polyurethanes chemically modified by octa(3-hydroxy-3-methylbutylmethylsiloxy) POSS (o-POSS) which varies from 2, 4, 6, 8 to 10 mass\%. These hybrid materials were tested by isothermal $\left(190{ }^{\circ} \mathrm{C}\right)$ and nonisothermal $\left(\beta=2,3.7,5\right.$ and $\left.10 \mathrm{~K} \mathrm{~min}^{-1}\right)$ chemiluminescence, and the thermal stability of gamma-radiation-aged samples was correlated with the change in the nanofiller loading and absorbed dose. The compositions where inorganic phase is less than $6 \%$ show an increasing thermal strength as o-POSS concentration enhances. The other samples with higher nanoparticle content present less stability in respect of inferior homologous composition. The nonisothermal chemiluminescence profiles are changing from one type of sample to the other where nanofiller induces different effects. The considerations on mechanistic aspects are discussed, too.
\end{abstract}

Keywords Polyurethane $\cdot$ POSS · Chemiluminescence $\cdot$ Thermal stability

\section{Introduction}

The hybrid materials are essential in all economical fields where the improved endurance is required. The enlarging knowledge areas of polyhedral oligomeric silsesquioxanes (POSS)-modified polymers have received deep attention [1-5] because the presence of this inorganic filler allows the manufacture of long-life engineering products. The diversity of polymer materials such as PMMA [6], silicone [7], epoxy resins [8] and polyurethanes [9] was studied, where POSS filler acts as a compound suitable for new resistant structures. These papers emphasize additive contribution to the polymer functionality. An intimate interaction between POSS nanoparticles and polyurethane structure was previously presented [10].

Traian Zaharescu

traian_zaharescu@yahoo.com

$\square$ Krzysztof Pielichowski

kpielich@pk.edu.pl

1 Department of Advanced Materials, INCDIE ICPE CA, 313 Splaiul Unirii, 030138 Bucharest, Romania

2 Department of Chemistry and Technology of Polymers, Cracow University of Technology, ul. Warszawska 24, 31-155 Kraków, Poland
The degradation of polyurethanes has been amply discussed because these materials have several applications in medical wear, chemical engineering, aircraft industry, nuclear areas $[9,11,12]$. The excellent radiation stability of polyurethanes [13] and their large processing dose range [14] recommends them for long-term applications. The degradation of polyurethanes accelerated by their exposure to high-energy radiation [14-16] occurs somewhat slowly because they show an evident tendency to cross-link [17]. The chemiluminescence (CL) examination on the thermal stability of polyurethane composite reveals the contribution of bond dissociation and the elimination of carbon dioxide to the evolution of thermal degradation in polyurethane matrices $[18,19]$. The spectroscopic (ATR-FTIR) analysis identified oxygenated products as main degradation products formed during natural and artificial aging of polyurethane foams [20].

The degradation mechanism of polyurethanes and their POSS composites was previously analyzed. The thermal stability study on the degradation of rigid polyurethanes foams modified with polyhedral oligomeric silsesquioxane on whose structure propanediolizobutyl or (3-hydroxy-3methylbutyldimethylsiloxy) moieties were grafted has pointed out the changes in physical and structural features 
caused by the interaction between polymer matrix and inorganic particles [12].

However, the studies on the stabilization of polyurethanes are scarcely published $[9,12,21]$. They underline the delay of oxidative degradation by the additive activities in respect of the scavenging free radicals, the efficient adsorption on particle surface or the penetration of radicals through channels existing in POSS morphology. The telechelic behavior of PU in the presence of modified POSS nanoparticles [22] confirms the remarkable thermal resistance of these compositions proving the further capacity of material for the oxidation prevention of creating radicals. The proofs on the improved endurance PU/ POSS systems are the results of phase stability investigation over large temperature range $\left(50-400{ }^{\circ} \mathrm{C}\right)$ [23].

In this paper, the stability investigations by chemiluminescence on radiation processes of MDI-based polyurethane modified with octa(3-hydroxy-3methylbutyldimethylsiloxy) polyhedral oligomeric silsesquioxane (o-POSS) are analyzed.

\section{Experimental}

\section{Synthesis materials}

Hybrid polyurethane/o-POSS materials were synthesized using 4,4'-diphenylmethane diisocyanate (MDI, SigmaAldrich), poly(tetramethylene glycol) (Invista), 1,4-butanediol (Sigma-Aldrich) and o-POSS (Hybrid Plastics) (Fig. 1) in a two-step process [10].

\section{Methods}

Radiation processing was accomplished in air at room temperature in an irradiation device (Ob Servo Sanguis, Hungary) provided with ${ }^{60} \mathrm{Co}$ source. The doses were 25 , 50 and $100 \mathrm{kGy}$. The dose rate was $1 \mathrm{kGy} \mathrm{h}^{-1}$. The samples were measured immediately after the end of each irradiation.

Isothermal and nonisothermal chemiluminescence spectra were recorded by means of LUMIPOL 3 (Slovak
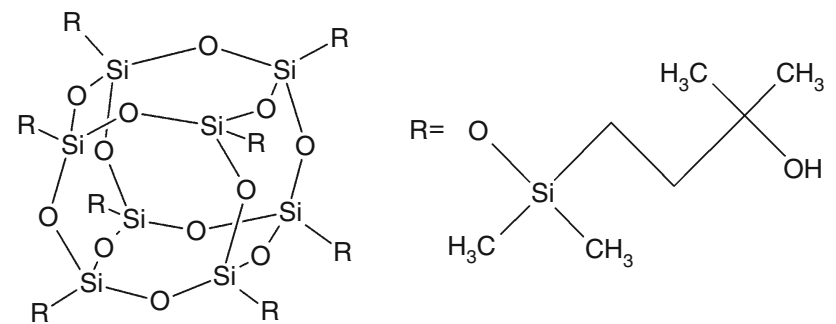

Fig. 1 Molecular configuration of octa(3-hydroxy-3-methylbutyldimethylsiloxy) polyhedral oligomeric silsesquioxane
Academy of Sciences, Bratislava). Thermal regimes were as follows: for isothermal investigations, the temperature was performed at 100,110 and $120{ }^{\circ} \mathrm{C}$, and for nonisothermal determinations the four heating rates, 2, 3.7, 5 and $10{ }^{\circ} \mathrm{C} \mathrm{min}{ }^{-1}$. Small square pieces weighing around $3 \mathrm{mg}$ were placed on aluminum pans which do not influence the oxidation profile.

\section{Results and discussion}

The radiation processing, which is an accelerated procedure for polymer modification, provides high concentration of reactive intermediates (free radicals as former entities). In the opposition with PP/POSS, PP being degraded by $\gamma$-irradiation in air [24], polyurethane/POSS hybrids exhibit higher oxidation resistance at small filler loadings [9]. As a result of polypropylene radiolysis, the values of decomposition temperature fall significantly down for low absorbed dose (less than $20 \mathrm{kGy}$ ), while the stability of polyurethane is improved for the concentration up to $4 \%$ [9].

The modification of POSS molecular configuration by the grafting of branched moiety brings about a supplementary effect on the efficiency in oxidation delay. The contribution of this kind of grafted POSS was previously reported for the promotion of cross-linking [25] via classical reactions, but high-energy exposure involves more intimately the changing amplitude. The molecular architecture would be defined by the competition between oxidation and radical scavenging [5]. If the substitute is not enough active for the slowing down degradation rate, polyurethane molecules are dominantly converted into alcohol by-products [26].

Isothermal CL investigation on pristine polyurethane shows curves with a minimum value (Fig. 2).

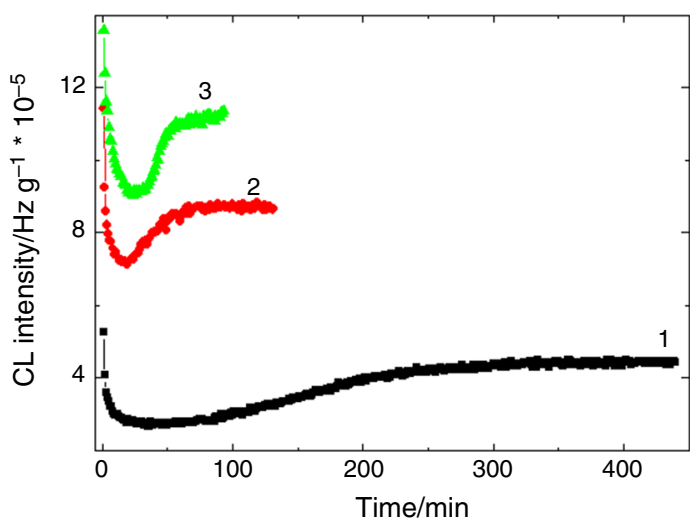

Fig. 2 Isothermal $\mathrm{CL}$ spectra recorded on pristine polyurethane samples. (1) $100{ }^{\circ} \mathrm{C}$, (2) $110{ }^{\circ} \mathrm{C}$ and (3) $120{ }^{\circ} \mathrm{C}$ 
They demonstrate fragmentation process accompanied by a slight oxidation on the descendant part followed by an advanced oxidation on the ascendant part of curves. An accelerated degradation is obtained as the working temperature increases because the quicker diffusion of oxygen feeds this process. The related mechanism was previously reported $[11,27]$. The temperature of $110{ }^{\circ} \mathrm{C}$ would be considered as an acceptable value for reliable experiments.

The presence of octa(3-hydroxy-3-methylbutyldimethylsiloxy)/polyhedral oligomeric silsesquioxane in the studied polyurethane matrix changes profoundly the shape of oxidation curves (Fig. 3).

The nonirradiated samples show CL curves with pronounced descending former part followed by an equilibrium plateau or slight monotone ascending portion. This last tendency is characteristic for pristine and $2 \%$ modified polyurethane, where the filler loading is minimal. It can be easy noticed that the increase in the o-POSS concentration improves significantly the oxidation strength of basic polymer till it reaches a reasonable figure $(6 \%)$. This feature was also found for polyurethane samples containing neat POSS [9], but the stability threshold was only $4 \%$. Although the content of filler becomes higher, up to $10 \%$ the sample stabilities remain higher than that was observed for pristine polyurethane. The $\mathrm{CL}$ curves recorded for superior concentrations ( 6 and $8 \%$ ) of o-POSS are placed in the upper region in respect of the most stable material $(6 \%)$ because the oxidation takes place faster. The highest content of o-POSS delays oxidation only on the first step of degradation. It would be explained by the saturation of o-POSS reacting positions; consequently, the competition between adsorption and oxidation of free radicals is gained by the oxidation aging.

The most suggestive comparison between the degradation of irradiated samples may be made on pristine and $6 \%$ loaded polyurethane (Fig. 4).

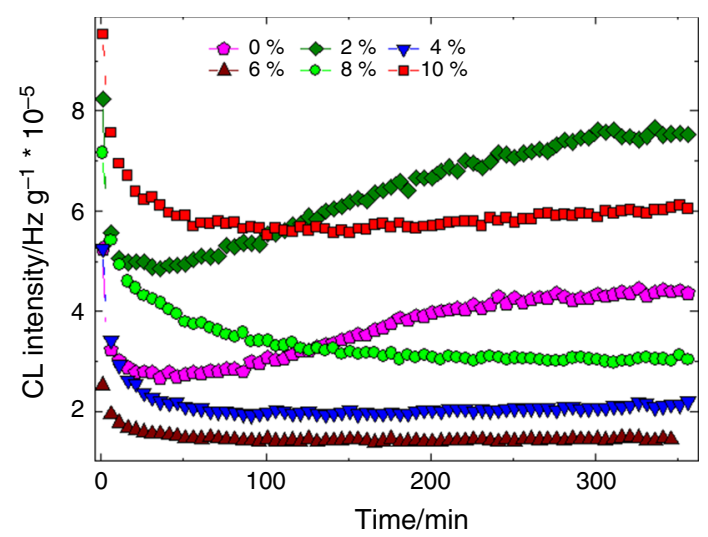

Fig. 3 Isothermal CL spectra recorded for all nonirradiated polyurethane hybrids. Testing temperature: $110{ }^{\circ} \mathrm{C}$
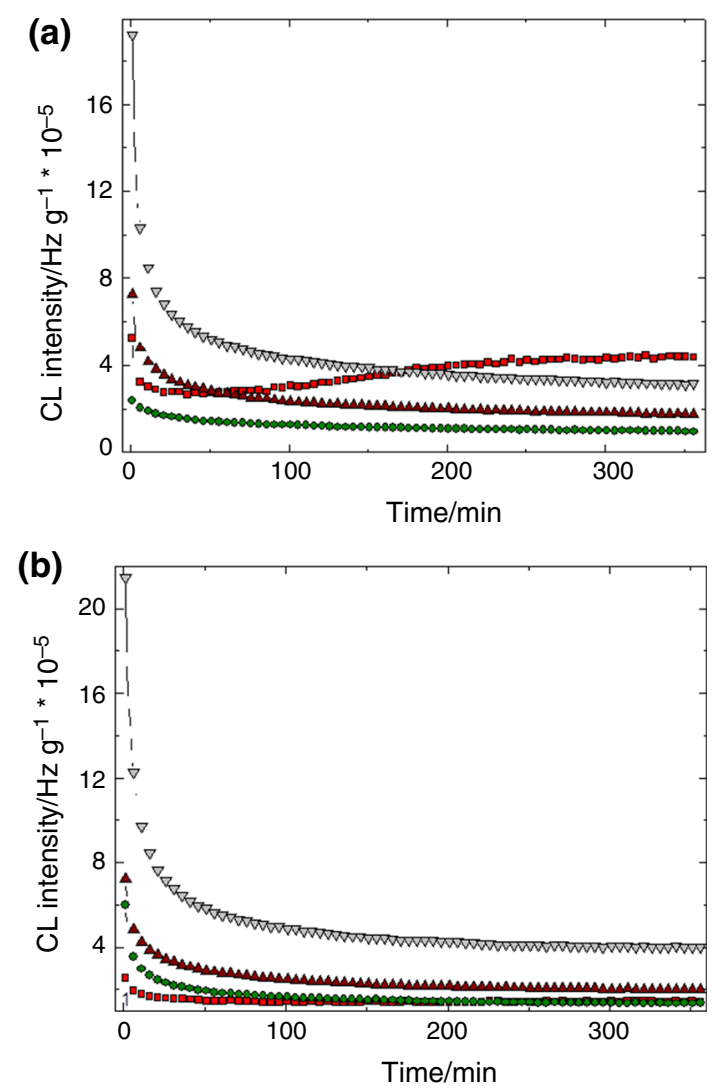

Fig. 4 Isothermal CL spectra recorded for neat (a) and 6\% o-POSS hybrid (b) polyurethane $\gamma$-irradiated at various doses. Testing temperature: $110^{\circ} \mathrm{C}$, (red square) $0 \mathrm{kGy}$, (green circle) $25 \mathrm{kGy}$, (dark red triangle) $50 \mathrm{kGy}$, (white inverted triangle) $100 \mathrm{kGy}$. (Color figure online)

In the case of pristine material, the irradiation causes a former stabilization at $25 \mathrm{kGy}$, while higher doses promote oxidation. The curve shapes over the first $10 \mathrm{~min}$ of oxidation are sharper in the case of modified polymer because the scavenging of free radicals is efficient. The higher CL intensities recorded on irradiated samples especially at $100 \mathrm{kGy}$ prove the availability of high radical concentration for oxidation in respect of unprocessed polymer. However, the values of CL intensities after the accomplished degradation tend to similar figures that characterize each filler concentration (Fig. 5).

The evolution of oxidation as the temperature increases is presented in Fig. 6.

On the low-temperature range (room temperature $-180^{\circ} \mathrm{C}$ ), the emission intensities are comparable because the concentration of free radicals is not high enough, at elevated temperatures, for example $250{ }^{\circ} \mathrm{C}$. The relative position of specific intensities confirms the behavior found by isothermal investigation. The neat polyurethane is the most unstable material, and the difference between pristine and compounded materials appears evidently during for advanced degradation state 


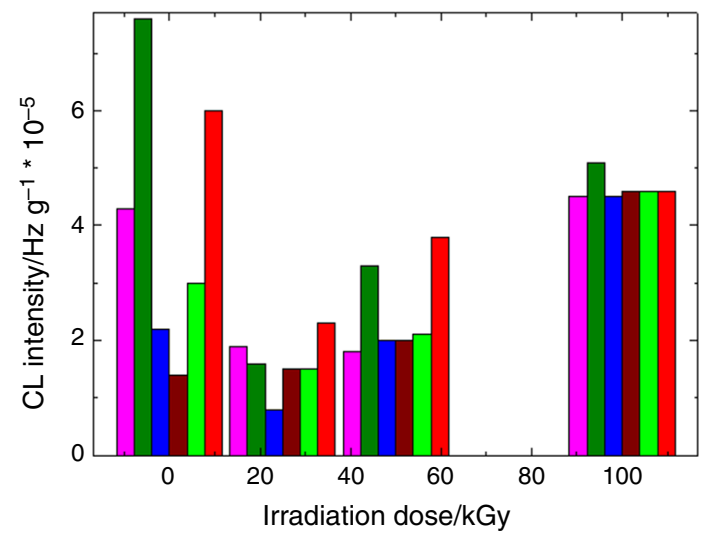

Fig. 5 Final values of CL emission intensities for oxidation of all polyurethane samples. The meaning of coloring is the same as in Fig. 3. (Color figure online)

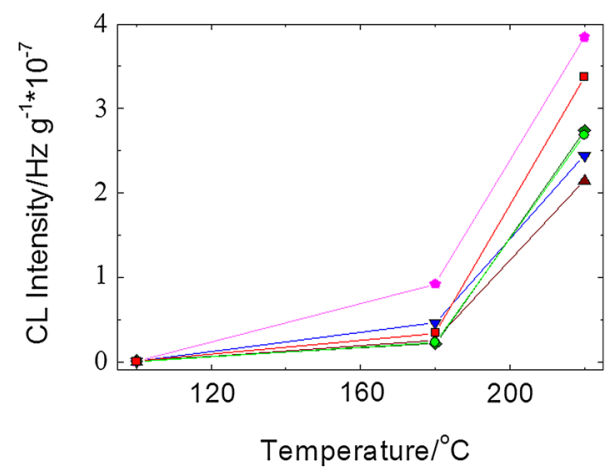

Fig. 6 Evolution of CL intensity for all unirradiated polyurethane samples at three temperatures. The meaning of coloring is the same as in Fig. 3. (Color figure online)

when the filler acts as intermediate scavenger. As it was previously reported [28], the degradation process occurs by the secondary peroxyl radicals which are further involved in different reactions like hydrogen and radical abstraction, disproportionation or partial recombination. Their feeding is practically delayed by o-POSS nanoparticles which retain the oxidation initiators.

The comparison of nonisothermal CL spectra for nonirradiated samples (Fig. 7) reveals the delay of oxidation by o-POSS nanoparticles.

This kind of materials reveals a limiting concentration threshold at $6 \%$, while the delimitation of protection regime in other engineering polymers like polyethylene, ethylene-propylene elastomer or any other thermoplastic material was never demonstrated.

The detailed analysis of the radiation degradation of polyurethanes based on infrared spectroscopy [15] where the tendency to cross-linking is proved by CharlesbyPinner representation and the degradation features is

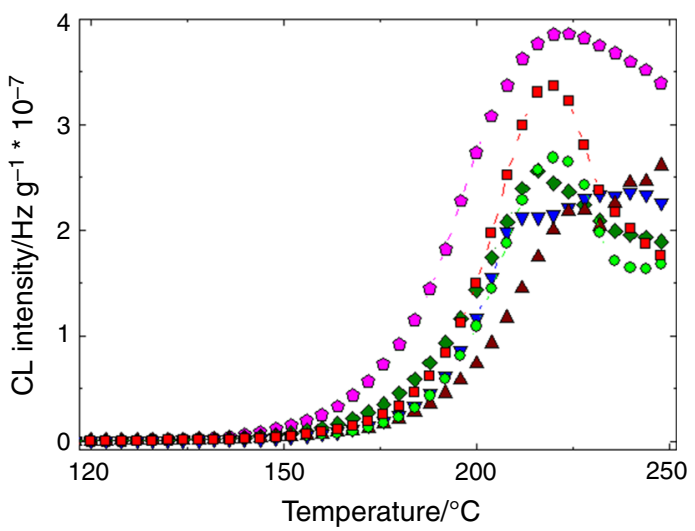

Fig. 7 Nonisothermal CL spectra recorded on all unirradiated polyurethane samples at heating rate $2{ }^{\circ} \mathrm{C} \mathrm{min}^{-1}$. The meaning of coloring is the same as in Fig. 3. (Color figure online)

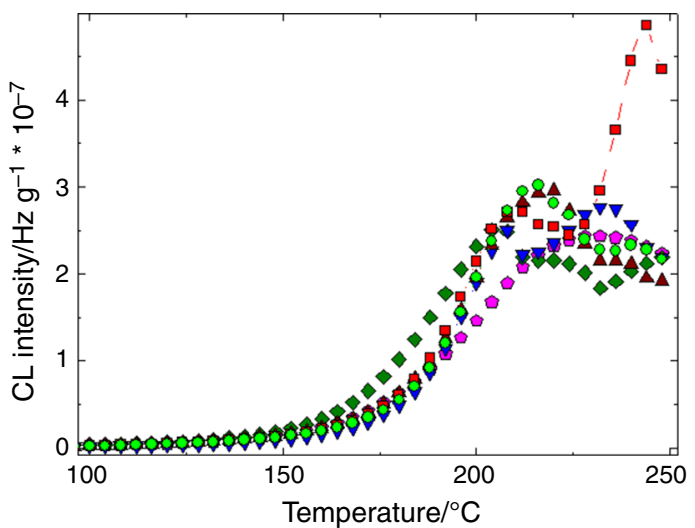

Fig. 8 Nonisothermal CL spectra recorded on all polyurethane samples irradiated at $25 \mathrm{kGy}$. Heating rate $2{ }^{\circ} \mathrm{C} \mathrm{min}{ }^{-1}$. The meaning of coloring is the same as in Fig. 3. (Color figure online)

justified by the changes in characteristic spectral absorption. The most sensitive parts of polyurethane molecules are found in amorphous zone as soft fragments and ethylene glycol moieties. In our cases, the scavenging activities shown by the polyurethane/o-POSS formulations irradiated at low dose $(25 \mathrm{kGy})$ characterizing the sterilization operation are similar on the low- and medium-temperature ranges and they do not strictly depend on material composition (Fig. 8).

The more intensive heating brings about an accelerated oxidation in the samples with medium amount of o-POSS. It may be ascribed to the availability of substituted octa(3hydroxy-3-methylbutyldimethylsiloxy) to hinder the detachment of scavenged intermediates due to its large volume.

The history of materials gets influence on the degradation manner by which polymer items are degrading. The irradiation and the features related to the radiochemical scission and cross-linking yields are permanently in competition during $\gamma$-exposures. The comparison between 

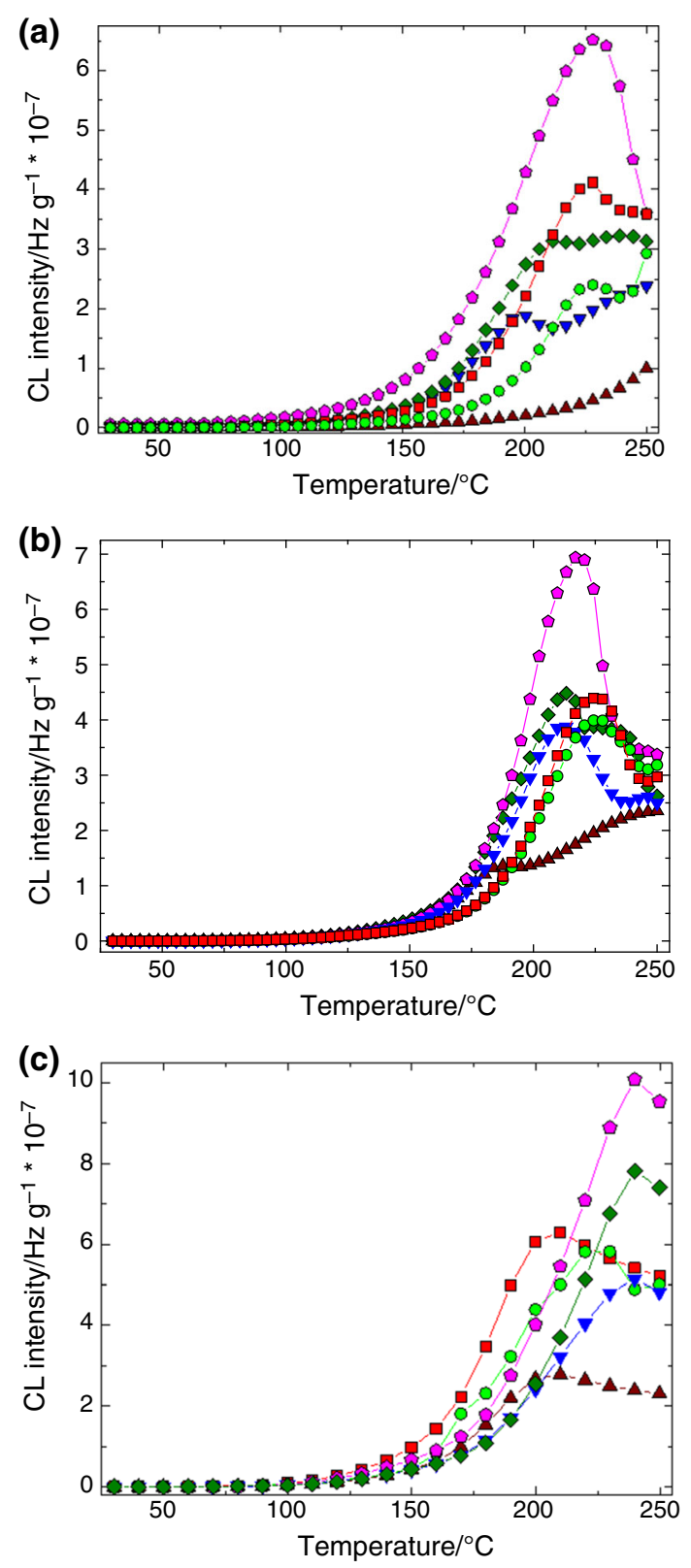

Fig. 9 Nonisothermal CL spectra recorded on all polyurethane samples measured in different conditions. a $50 \mathrm{kGy}, b=5{ }^{\circ} \mathrm{C} \min ^{-1}$, b $100 \mathrm{kGy}, b=3.7^{\circ} \mathrm{C} \min ^{-1}$ and c $100 \mathrm{kGy}, b=10^{\circ} \mathrm{C} \mathrm{min}-1$. The meaning of coloring is the same as in Fig. 3. (Color figure online)

Figs. 8 and 9a, higher emission intensities for longer exposure of polyurethane/o-POSS samples, can be accounted, but the likeness of each composition for the two doses does not confirm similarity in the progress of degradation.

The differences in the radical concentrations and in the filler loadings cause the shift of intensity peaks toward higher temperatures. The simultaneous molecular fragmentation, radical scavenging by filler and their reactions change the oxidation profile. The most relevant behavior for the both situation is the preservation of stability ranking.

Figure 9b, c illustrates the thermal behavior of similarly irradiated samples by CL measurements at two different heating rates. The higher the testing rate, the greater the emission intensities. The most stable composition is polyurethane $/ 6 \%$ o-POSS which shows this favorable characteristic even at higher temperatures. The analysis of intimate mechanism of this material response leads to the conclusion that this filler content corresponds to a saturation threshold for radical scavenging. Further, the increased o-POSS amount does not sustain superior stability; by contrary, the oxidation progresses faster because of the greater concentration of blocked initiators.

\section{Conclusions}

The thermal behavior of polyurethane/octa(3-hydroxy-3methylbutyldimethylsiloxy)/polyhedral oligomeric silsesquioxane presents a stability threshold at the concentration of o-POSS of $6 \%$. The contribution of this filler in the nanosize state in polyurethane matrix is based on its availability for the scavenging radicals formed by molecular fragmentation. Similar stability limit was also shown by polyurethane loaded with pristine POSS, but the highest stability was noticed at $4 \%$. The investigations accomplished by isothermal and nonisothermal chemiluminescence revealed the increase in thermal and radiation stabilities up to limit concentration followed by the more accelerate oxidation. The prented results are useful for the assessment of polyurethane based medical wear which have to resist over long operation term. The accelerated degradation carried out under $\gamma$-irradiation is a proper manner to demonstrate the availability of these hybrid materials for nuclear application.

Acknowledgements Authors (EH and KP) are grateful to the National Science Center in Poland for financial support under Contract No. DEC-2011/02/A/ST8/00409.

Open Access This article is distributed under the terms of the Creative Commons Attribution 4.0 International License (http://crea tivecommons.org/licenses/by/4.0/), which permits unrestricted use, distribution, and reproduction in any medium, provided you give appropriate credit to the original author(s) and the source, provide a link to the Creative Commons license, and indicate if changes were made.

\section{References}

1. Pielichowski K, Njuguna J, Janowski B, Pielichowski J. Polyhedral oligomeric silsesquioxane (POSS)-containing nanohybrid polymers. Adv Polym Sci. 2006;201:225-96. https://doi.org/10. 1007/12_077. 
2. Wu J, Mather PT. POSS polymers: physical properties and biomaterials applications. J Macromol Sci Part C: Polym Rev. 2009;49:25-63. https://doi.org/10.1080/15583720802656237.

3. Madbouly SA, Otaigbe JU. Recent advances in synthesis, characterization and rheological properties of polyurethanes and POSS/polyurethane nanocomposites dispersions and films. Prog Polym Sci. 2009;34:1283-332. https://doi.org/10.1016/j.progpo lymsci.2009.08.002.

4. Kuo S-W, Chang S-C. POSS related polymer nanocomposites. Prog Polym Sci. 2011;36:1649-96. https://doi.org/10.1016/j. progpolymsci.2011.05.002.

5. Zhang W, Müller AHE. Architecture, self-assembly and properties of well-defined hybrid polymers based on polyhedral oligomeric silsesquioxane. Prog Polym Sci. 2013;38:1121-62. https:// doi.org/10.1016/j.progpolymsci.2013.03.002.

6. Xu HG, Yang BH, Wang JF, Guang SY, Li C. Preparation, $\mathrm{T}_{\mathrm{g}}$ improvement, and thermal stability enhancement mechanism of soluble poly(methyl methacrylate) nanocomposites by incorporating octavinyl polyhedral oligomeric silsesquioxanes. J Polym Sci Polym Chem. 2007;45:5308-17. https://doi.org/10.1002/pola. 22275.

7. Joshi V, Srividhya M, Dubey M, Ghosh AK, Saxena A. Effect of functionalization on dispersion of POSS-silicone rubber nanocomposites. J Appl Polym Sci. 2013;130:92-9. https://doi. org/10.1002/app.39112.

8. Ramirez C, Rico M, Barral L, Diez J, García-Garabal S, Montero B. Organic/inorganic hybrid materials from an epoxy resin cured by an amine silsesquioxane. J Therm Anal Calorim. 2007;87:69-72. https://doi.org/10.1007/s10973-006-7819-4.

9. Zaharescu T, Pielichowski K. Stabilization effects of POSS nanoparticles on gamma-irradiated polyurethanes. J Therm Anal Calorim. 2016;124:767-74. https://doi.org/10.1007/s10973-0155191-y.

10. Raftopoulos KN, Koutsoumpis S, Jancia M, Lewicki JP, Kyriakos K, Mason HE, Harley SJ, Hebda E, Papadakis CM, Pielichowski $\mathrm{K}$, Pissis P. Reduced phase separation and slowing of dynamics in polyurethanes with three-dimensional POSS-based cross-linking moieties. Macromolecules. 2015;48:1429-41. https://doi.org/10. 1021/ma5023132.

11. Jipa S, Zaharescu T, Setnescu R, Ciobanu C, Caşcaval CN. Chemiluminescence study on the thermal stability of $\mathrm{Er}^{3+}$-doped poly(urethane-lactate). J Optoelectr Adv Mater. 2009;9:2763-8.

12. Albu P, Bolcu C, Vlase G, Doca N, Vlase T. Kinetic of degradation under non-isothermal conditions of a thermooxidative stabilized polyurethane. J Therm Anal Calorim. 2011;105:685-9. https://doi.org/10.1007/s10973-011-1497-6.

13. Makuuchi K, Cheng S, editors. Radiation processing of polymers materials and its industrial applications. Hoboken: Wiley; 2012. p. 232.

14. Clough RL. Radiation-resistant polymers. In: Encyclopedia of polymer science, 2nd ed., vol. 13. New York: Wiley; 1998. p. $667-708$.

15. Adem E, Angulo-Cervera E, González Jiménez A, Valentín JL, Marcos-Fernández A. Effect of dose and temperature on the physical properties of an aliphatic thermoplastic polyurethane irradiated with an electron beam. Radiat Phys Chem. 2015;112:61-70. https://doi.org/10.1016/j.radohyschem.2015.03. 017.
16. Ravat B, Grivet M, Chambaudet A. Evaluation of the degradation and oxidation of polyurethanes versus the electron irradiation parameters: fluence, flux and temperature. Nucl Instrum Methods Phys Res. 2001;179:243-8. https://doi.org/10.1016/S0168583X(01)0057-7.

17. Assink RA. Radiation crosslinking of polyurethanes. J Appl Polym Sci. 1985;30:2701-5. https://doi.org/10.1002/app.1985. 070300634.

18. Rychlý J, Lattuati-Derieux A, Lavédrine B, Matisová-Rychlá L, Maliková M, Csomorová K, Janigová I. Assessing the progress of degradation in polyurethanes an thermal analysis. II. Flexible polyether- and polyester-type polyurethane foams. Polym Degrad Stab. 2001;96:462-9. https://doi.org/10.1016/j.polymdegradstab. 2011.01.012.

19. Maliková M, Rychlý J, Matisová-Rychlá L, Csomorová K, Janigová I, Wilde H-W. Assessing the progress of degradation in polyurethanes by chemiluminescence. I. Unsaturated polyurethane films. Polym Degrad Stab. 2010;95:2367-75. https://doi. org/10.1016/j.polydegradstab.2010.08.016.

20. Lattuati-Derieux A, Thao-Heu S, Lavédrine B. Assessment of the degradation of polyurethane foams after artificial and natural ageing by using pyrolysis-gas chromatography/mass spectrometry and headspace-solid phase microextraction-gas chromatography/mass spectrometry. J Chromatogr. 2011;A1218:4498-508. https://doi.org/10.1016/j.chroma.2011.05.013.

21. Thapliyal BP, Chandra R. Advances in photodegradation and stabilization of polyurethanes. Prog Polym Sci. 1990;15:735-50. https://doi.org/10.1016/0079-6700(90)90010-X.

22. Tsukahara Y, Adachi K. Telechelic polymers: preparation and application. Encycl Polym Nanomater. 2014. https://doi.org/10. 1007/978-3-642-36199-9_201-1.

23. Raftopoulos KN, Jancia M, Aravopoulou D, Hebda E, Pielichowski K, Pissis P. POSS along the hard segments of polyurethanes. Phase separation and molecular dynamics. Macromolecules. 2013;46:7378-86. https://doi.org/10.1021/ ma401417t.

24. Choi J-H, Jung C-H, Kim D-K, Nho Y-C, Kang P-H, Ganesan R. Preparation of polymer/POSS nanocomposites by radiation processing. Radiat Phys Chem. 2009;78:517-20. https://doi.org/10. 1016/j.radphyschem.2009.03.037.

25. Zheng L, Hong S, Cardoen G, Burgaz E, Gido SP, Couglin EB. Polymer nanocomposites through controlled self-assembly of cubic silsesquioxane scaffolds. Macromolecules. 2004;37:8606-11. https://doi.org/10.1021/ma048557.

26. Harris DJ, Assink RA, Celina M. NMR analysis of oxidatively aged HTPB/IPDI polyurethane rubber: degradation products, dynamics and heterogeneity. Macromolecules. 2001;34:6695-700. https://doi.org/10.1021/ma0108766.

27. Roşu D, Roşu L, Mustaţă L, Varganici C-D. Effect of UV radiation on some semi-interpenetrating polymer networks based on polyurethane and epoxy resins. Polym Degrad Stab. 2012;97:1261-9. https://doi.org/10.1016/j.polymdegradstab. 2012.05.035.

28. Fratricová M, Šimon P, Schwarzer H-WW. Residual stability of polyurethane automotive coatings measured by chemiluminescence and equivalence of Xenotest and Solisi ageing tests. Polym Degrad Stab. 2006;91:94-100. https://doi.org/10.1016/j.poly mdegradstab.2005.04.025. 